(i) ${ }_{\text {в }}$

\title{
ESTRUCTURA Y SUJETO DEL INCONSCIENTE: LA FICCIÓN DE LO SIMBÓLICO
}

\author{
Subconscious Structure and Subject: The Fiction of what Is Symbolic
}

Estrutura e sujeito do inconsciente: a ficção do simbólico

es

\section{RESUMEN}

Hablar de epistemología en psicoanálisis implica un doble trabajo: el de encontrar los vestigios en la teoría analítica, preferentemente en los aportes de Freud y Lacan, y el de revisar fuera del contexto de la disciplina los conceptos con que se alimenta. En muchas ocasiones, estos conceptos provienen de diferentes orillas del conocimiento, lo cual implica un esfuerzo adicional de dar su lugar a cada una de las nociones, específicamente para notificar lo que sucede en esos dos espacios cruciales para el psicoanalista, la clínica y la cotidianidad. En este orden de ideas, este trabajo trae a colación dos conceptos muy importantes para las actividades comprometidas con la clínica y la cotidianidad: las nociones de 'estructura'y 'sujeto' en psicoanálisis, así como su relación y alcance.
Evaristo Peña Pinzón (Colombia)

Magíster en Psicoanálisis, Subjetividad y Cultura

Universidad Antonio Nariño

evpena@uan.edu.co

\section{en}

\section{ABSTRACT}

When talking about epistemology in psychoanalysis, we deal with two things: finding the remains of the analytical theory -especially with respect to Freud and Lacan- and checking out-of-context concepts it uses. Frequently, these concepts come from different sources. This implies an additional effort to give notions their rightful place, specifically when reporting what happens in these two spaces (essential for a psychoanalyst, the clinic, and routine.) Therefore, this article presents two concepts that are important for the activities related to clinic and routine: the notions of structure and subject in psychoanalysis, their relationship, and scope.

KEYWORDS: psychoanalysis, structure, unconscious, subject, clinic.

\section{RESUMO}

Falar de epistemologia em psicanálise implica um duplo trabalho: o de encontrar os vestígios na teoria analítica, preferentemente nos aportes de Freud e Lacam, e o de revisar fora do contexto da disciplina os conceitos com que se alimenta. Em muitas ocasiões, estes conceitos provêm de diferentes extremos do conhecimento, o qual implica um esforço adicional de dar o seu lugar a cada uma das noções, especificamente para notificar o que sucede em esses dois espaços cruciais para o psicanalista, a clínica e a cotidianidade. Em este ordem de ideias, este trabalho traz a colação dois conceitos muito importantes para as atividades comprometidas com a clínica e a cotidianidade: as noções de estrutura e sujeito em psicanálise, assim como a sua relação e alcance.

PALAVRAS CHAVE: : psicanálise, estrutura, inconsciente, sujeito, clínica. 


\section{FUNCIÓN DE LA NOCIÓN DE 'ESTRUCTURA’EN PSICOANÁLISIS}

Boudon (1972) ofrece en su trabajo una idea de cómo fue el fenómeno ocurrido con Lacan y su seminario. Lacan fundó un estilo propio, suyo, digno de amores y odios acérrimos, que le valió tener un "ejército" de interesados en el psicoanálisis en un determinado momento, justo luego disolvería esa escuela que nunca se solidificó totalmente, y dejó en claro que las condiciones para la transmisión del análisis no podían ser de cualquier forma, pues se corría el riesgo de banalizarlo. La labor de Lacan se funda en una premisa: la de leer a Freud a la letra (Lacan, 2000c, p. 121), con lo cual convierte este método en una política para trabajar en psicoanálisis, así como en una manera de construir una forma particular de transmitir su enseñanza, la de $s u$ lectura de Freud, enseñanza lacaniana que está implicada en la contemporaneidad revuelta de las años en que perduró. Hoy podemos preguntarnos cómo lo asumimos, cómo cada interesado en el psicoanálisis asume las condiciones que los padres de la disciplina propusieron y cómo nos adjuntamos o no en instituciones psicoanalíticas. Para no ir muy lejos con este tema, que amerita otro comentario aparte, sobra decir que la condición de condiciones para cada interesado o interesada es hacer un análisis, con un psicoanálista, y llevarlo lo más lejos posible.

Dicho esto, el ámbito en el que se creó y se recreó el psicoanálisis es el que favorecerá el uso de algunos recursos prestados de otras disciplinas. Este es el caso de muchos conceptos freudianos, pero más evidente es el caso de la noción de 'estructura', la cual se formaliza como concepto restrictivo a partir del trabajo de Lacan para pensar las vicisitudes del psicoanálisis.

Lo específico del modo de proceder de Lacan se encuentra en que, más allá de una moda conceptual, existe un método para él, que se alimenta históricamente de las elaboraciones principales de Freud, a saber: sus conceptos fundamentales, forjados en la experiencia clínica y en el esfuerzo de teorización al principio del psicoanálisis, que indican un camino muy importante, como el mismo Lacan dijera de Freud, procediendo como arqueólogos, con lo que dicen los pacientes y con la misma obra del fundador del psicoanálisis, pues Freud desenterró los monumentos que ahora es tarea nuestra volver a leer, ojalá en el sitio donde ocurrió el desentierro. Esta metáfora, aun si es imprecisa, permite indicar cuál es el método de elaboración que realizamos con los conceptos, con las viñetas clínicas y con los casos que Frued legó, asumiendo toda su producción como un hallazgo que sorprendió a su descubridor, y frente al cual él supo conservar las condiciones y articulaciones fidedignas para que luego Lacan, y posteriormente nosotros, nos acerquemos a hacer su lectura, con sorpresivos encuentros. Esto justifica volver siempre a Freud, y ojalá con la linterna de la lectura lacaniana, que abre dimensiones de comprensión adicionales gracias a su capacidad para discernir y teorizar, inventando con nuevas nociones, lo que del inconsciente se revela.

Con Lacan se trata de una retrospectiva, de una lectura que hace hablar en Freud conceptos creados por el primero, desarrollando y articulando otras nociones que, sin ser necesariamente explícitas en Freud, se considerarán en adelante como parte de su "espíritu"; complejidad de elementos que se retoman con una lectura más que renovada, distinta, que desea adelantar claridades ahí donde se produjeron oscuridades.

Así, existe una retrospectiva a la que invita Lacan: revisar el texto de Freud a la letra, prestando oídos a los fenómenos de sentido de la palabra tal como el propio Freud propuso ante las diferentes formaciones del inconsciente con que se encontraba para sorprendernos, por ejemplo, con la capacidad generativa propia del lenguaje, y así, posteriormente, poder consolidar una teoría sobre el significante. En este sentido, los trabajos freudianos sobre "El mecanismo psíquico de la desmemoria" (Freud, 1898/2004), "Los recuerdos encubridores" (Freud, 1899/2004) y "La interpretación de los sueños" (Freud, 1900/2004) demuestran los elementos cruciales de la precisión del significante que opera en la formación del inconsciente en las condiciones que Lacan (2000) declarará de "estar estructurado como un lenguaje”. En otras palabras, Freud sigue siendo vigente, lo cual es así es porque logró evidenciar los procesos y elementos determinantes del psiquismo, aun si en su momento le hicieron falta conceptos, que no estaban a su alcance por el incipiente desarrollo de otras disciplinas y la propia, creada palmo a palmo por él.

Para comprender lo que se puede comprender, debemos estar contextualizados respecto del recorrido realizado por Freud. En un primer momento, presenta sus trabajos como neurólogo, explicando la sintomatología por medio de un sistema psíquico propio de las ideas 
Evaristo Peña

Pinzón I

Panorama I

pp. $130-137 \mid$

Volumen 9 |

Número 16 |

Enero-junio I

2015 |
Un tercer momento será el nominado como segunda tópica. Freud (1923/2004) usa en este los conceptos 'ello', 'yo', 'superyó' como una propuesta más sistemática sobre la constitución del psiquismo humano, con lo cual

\begin{abstract}
uno se forma entonces la representación de que dos fuerzas psíquicas han participado en la producción de estos recuerdos [diremos formaciones del inconsciente] [...] Estas dos fuerzas de contrapuesto efecto no se cancelan entre sí; tampoco sucede que un motivo avasalle al otro - con o sin menoscabo-, sino que sobreviene un efecto de compromiso, algo análogo a la formación de una resultante dentro del paralelogramo de fuerzas (Freud, 1899/2004, pp. 300-301).
\end{abstract}

Pasarán dieciséis años y una elaboración permanente para que se publique el artículo central que expresa la primera tópica freudiana en toda su extensión, el dedicado a "Lo inconsciente" (Freud, 1915/2004).$$
\text { cado "Lo inconsciente" (Freud, 1915/2004). }
$$

"Proyecto de psicología" (Freud, 1895/2004): toda una novedad que introduce una psicología para comprender aquello que está más allá de lo orgánico, todo un asunto nuevo, descubierto y susceptible de tratamiento especítirá progresivamente en el campo clínico psicoanalítico. En este momento, Freud piensa los procesos anímicos sin desprenderse del todo de la idea biológica del organismo humano (Freud, 1895/2004); por ende, encontraremos un ternario de conceptos que hacen referencia
al principio del placer, el principio de constancia y el principio de realidad, así como una referencia al lugar enfermar para poder cumplir con dicho deber. Su explicación es clara desde antes de la época definida como primera tópica, que va de 1900 a 1920:

logra, de manera magistral, no tener que reescribir toda la elaboración realizada hasta el momento: lo que hace es estratificarla, la complejiza en el sentido de introducir con mayor delicadeza elementos que no se han tenido en cuenta hasta entonces. También es cierto que él mismo confiesa que si es necesario cambiar elementos de la teoría en beneficio de mejores explicaciones y de mayor eficacia en el tratamiento, el científico no debe tener miramientos con su producción. Así procederá, y dejará algunos temas sin conclusión, mientras explorará unos, los fundamentales, con mayor atención y dedicación.

Volviendo a los tres instantes de la producción freudiana, es evidente que el autor constituye siempre ternarios funcionales y explicativos, que serán base de otros sistemas que, en cuanto nociones teóricas, explican su funcionamiento como constitutivos y constituyentes del acaecer psíquico. De las mayores evidencias de esto, son las nociones derivadas del complejo de Edipo y el complejo de castración, entre otros. A efectos de nuestra discusión, se debe tener en cuenta que la naturaleza de los conceptos y de los elementos propuestos por Freud no son unidimensionales, sino que comprometen en muchos casos la multifuncionalidad, producto del esfuerzo de la teorización a partir de la clínica y la necesidad de componer un andamio explicativo sólido, sustentado siempre en la práctica, pensando principalmente en la contradicción, o lo paradójico, que ella expone.

Si bien es cierto que Freud abandona algunas de sus conceptualizaciones, también es verídico que las ideas fundamentales, como la del funcionamiento del aparato psíquico en esfuerzo por una interdependencia de sistemas, subsistirán y lograrán salir adelante, comprobadas en la práctica clínica, generando aciertos y éxitos en las curas. Asi mismo, subsiste la manera como construye su metodología, la de un progresivo análisis, deconstructivo podemos decir hoy, tanto de los síntomas y enfermedades de sus pacientes como de la propia revisión y ajuste de la teoría.

Llegamos entonces al puerto de hablar de la estructura. Lacan retomará este método y, con la reciente creación y uso para él de la noción de 'estructura', restablecerá la validez de los conceptos freudianos, dándoles un lugar de organización fundamental, acudiendo a la luz que brindan las nuevas posturas de diversas disciplinas, 
entre ellas principalmente la lingüística estructural y los avances en la antropología, de las que echará mano para que su nueva construcción se sostenga.

En Lacan la noción de 'estructura cobrará relevancia con la condición de volver a los sistemas explicativos de Freud, que comprometen:

- El funcionamiento de los elementos implicados en la constitución del psiquismo para el ser humano, pensándolos como mínimos irreemplazables.

- La interdependencia de los elementos en cuanto dinámicos; ya no pensando la geografía de lo fisiológico, sino el dinamismo de las relaciones que generan compromiso entre instancias.

- Las congruencias y oposiciones existentes en los sistemas y en relación con otros sistemas. Esta situación lleva a reconocer las organizaciones, sus elementos y las oposiciones que se presentan, generando lógicas estructurales.

- Todo lo anterior teniendo siempre en cuenta que existe la falla, la cual debe ser entendida como imposibilidad de totalidad, imposibilidad de homeostasis, imposibilidad de integración absoluta. En la realidad experimentada por el individuo, existe la condición de que se trata de una ficción (Gerber, 1997), que el Otro ${ }^{1}$ está en falta, está destituido por lógica de un lugar de absoluto, y esto es el centro del asunto freudiano.

Además, pensar desde la noción de 'estructura' será pilar de un compromiso metodológico que distingue al psicoanálisis de otras disciplinas que crean saber sobre lo humano, sobre todo las que tienen metas terapéuticas, tanto por su objeto de estudio como por la técnica empleada en la clínica, ambas distantes, en el psicoanálisis, de las demás aproximaciones investigativas. En este punto se hace necesario reconocer que el psicoanálisis no abandona la rigurosidad científica, por lo cual ella es su norte desde que Freud comenzó el andamiaje de su teoría. Vale la pena revisar la forma como construye la investigación realizada sobre los sueños (Freud, 1900/2004).

La noción de 'estructura' entonces, con sus sinónimos (patrones-relaciones-sistemas), hace juego con el psicoanálisis en cuanto se parte de la contextualización de sus conceptos y de su funcionamiento tanto en lo práctico como en lo teórico, y específicamente partiendo de la estructura del lenguaje, en el que cada significante

1 Otro, concepto con el cual Lacan nombra el lugar de lo simbólico, el tesoro de lo simbólico, que antecede al sujeto y condiciona la estructura de significantes que darán lugar a cada nuevo ser humano en las redes del deseo, del deseo del Otro. remite a otros significantes para construir sentido. Así, la función de estructura solo es viable partiendo de una lectura actual en retrospectiva del rescate de la experiencia de Freud, método propuesto por Lacan, que promueve la función creadora de nuevos conceptos, siempre que sean en concordancia con la experiencia. Con sorpresa, entonces, leemos en la nuez del psicoanálisis el tema propiamente humano: somos seres de lenguaje.

\section{¿QUÉ ES ESTRUCTURA EN EL PSICOANÁLISIS?}

El psicoanálisis no define como tal lo que significa estructura para sí mismo o dentro de su cuerpo de saber. Pero sí enunciará qué de ese cuerpo teórico corresponde a la noción de 'estructura y qué no, partiendo de lo que la experiencia clínica revela. Es decir, es pasando por la cuestión de la psicopatología en psicoanálisis, que lejos de designar un modo de enfermedad al estilo médico, designa modos lógicos y particulares frente al complejo de Edipo, el deseo, la castración, el goce y el síntoma. Estas nociones recién escritas son las que permiten a un practicante de la disciplina ubicarse con la teoría en la práctica y con una técnica ética en función de las vicisitudes del deseo de su paciente.

Para el psicoanálisis el lenguaje es estructura. De la misma manera, el inconsciente lo es y asimismo el sujeto de la falta, el sujeto del inconsciente. Aquí surgen preguntas: ¿qué es el sujeto para el psicoanálisis? ¿Cuál es el papel del lenguaje para el sujeto? ¿Solo hablamos de sujeto del inconsciente, y por qué? ¿De qué hablamos cuando no se habla del sujeto del inconsciente, por ejemplo cuando se habla del yo o del individuo?

Lacan dirá en varios momentos su aforismo "el inconsciente está estructurado como un lenguaje", haciendo hincapié ahora en un punto, luego en otro. Dediquemos un instante a preguntar por el estatuto estructural del inconsciente. El punto clave es la cuestión del encuentro freudiano con las investiduras de las palabras como modelo de memorización del cuerpo humano, idea que retomará el mismo Freud progresivamente hasta que Lacan complejiza el asunto con las ideas traídas de la lingüística estructural, a partir de los conceptos 'significante' y 'significado', derivados de las explicaciones de De Sausurre (1945), para dar cuenta de la construcción del sentido en el ser humano.
Estructura

y sujeto del

inconsciente:

la ficción de lo 
En Freud encontraremos estos puntos de amarre, el significante y el significado, en relación, por ejemplo, con el síntoma y otras formaciones del inconsciente de las que es posible hacer una traducción: si el síntoma, el sueño, el lapsus, son traducibles, es en cuanto que estos fenómenos poseen un particular ordenamiento que es posible descifrar. Un ejemplo fehaciente es el indicado sobre el malestar de una mujer joven aquejada de accesos de vómito: "A menudo habíamos comparado (Breuer y yo) la sintomatología histérica con una escritura figural que, tras descubrir algunos casos bilingües, atinábamos a leer. En ese alfabeto, vómito significa asco" (Freud, 18931895/2005, pp. 144-145).

Entonces, existe estructura. Por eso Freud propone, de la clínica y la "Psicopatología de la vida cotidiana" (Freud, 1901/2005), la posibilidad de estudiar los fenómenos psíquicos con conceptos y métodos de la teoría literaria de su época, con los modos particulares como se procede para la construcción de las ideas: la condensación y el desplazamiento como modelos del actuar autónomo del inconsciente para revelarse al yo, burlando su censura. Lacan lo seguirá y retomará el método acudiendo a la lingüística, con los conceptos de 'metáfora' y 'metonimia' (Lacan, 2000).

Freud dirá que el material de los sueños, como el de los síntomas, procede de una particular simbolización, de ahí que el sistema de investigación y develamiento de sentidos y relaciones por él creado se fundamente en hechos de palabra, los cuales son la forma como se muestra el inconsciente, en la particular cotidianidad de los pacientes y, en general, en el ser humano. Freud (1900/2004) determina así que la existencia de los fenómenos mencionados corresponde a lo que realmente es psíquico, tras lo cual queda lo inconsciente en equivalencia con lo que verdaderamente es la realidad psíquica humana. Ahora, esto solo puede suceder en cuanto, para su establecimiento, se requieren los elementos ofrecidos por el lenguaje y agenciados mediante la relación primordial con un semejante que salve de la indefensión primordial en la que vive el neonato (Freud, 1895/2004), lo cual es "lo propiamente humano, es la cuestión que nos distingue de los demás animales”. Si el Otro, agente de rescate de la indefensión original, es eficaz, lo es por un asunto de lenguaje, de estructura fundamental ficcional, humana, no por una condición instintiva o natural, que en realidad no opera en la condición humana desde que se hace uso del lenguaje como rector de la realidad.
Vemos los juegos de relaciones que se trazan en la teorización: el lenguaje, la palabra y el Otro son elementos fundamentales en la construcción de los síntomas y de los demás fenómenos inconscientes, susceptibles de recuerdo, de repetición y de traducción, justamente porque comparten su funcionamiento como un lenguaje, desde el mismo entramado de constitución subjetiva. Lacan irá más allá y dirá entonces que lo inconsciente está estructurado como un lenguaje justo por las maneras, por el modo particular de funcionamiento, proponiendo que este es causa y consecuencia al mismo tiempo para el individuo humano. El lenguaje es el medio por el cual se estructura el ser humano, por lo cual una de sus consecuencias es ser el vínculo de los demás seres hablantes. Cuando esto no sucede, porque puede no suceder, tendremos que hablar de una estructuración diferente, fuera de las reglas del complejo de Edipo y la castración, y más comprometidas con una formalización alrededor del goce y del síntoma que cuestiona la existencia del sujeto en la cadena significante; he aquí la diferencia entre las estructuras neuróticas y psicóticas de la subjetividad.

En Lacan la organización particular de sus teorías lo llevarán a designar que la manera como se causa el sujeto es estructural, gobernada por una serie de elementos que son necesarios, irreductibles, y que siempre forjan una falla en la constitución subjetiva. Aquí se debe hacer la claridad de que no es posible la ausencia de falla, es más bien que existen modos particulares, subjetivos, de estructurar la realidad a la sazón de los elementos de base que, en pocas palabras, en cuanto mediados por el lenguaje y el deseo del Otro siempre implican insatisfacción, desfase para el sujeto, que siempre se encontrará frente a una ficción provista por lo simbólico (Gerber, 1997).

Entonces, la función de estructura quiere decir en psicoanálisis los juegos y las mediaciones simbólicas, imaginarias y reales, que son convocadas en la experiencia de un individuo para que se cifren las maneras particulares de enfrentarse el deseo, el síntoma y el goce, lo cual en ocasiones tiene o no los elementos del complejo de Edipo y de la castración. De ahí que esta definición nos aproxime a la cuestión de las estructuras psicopatológicas, leídas como modos particulares de tramitar la castración y los significantes primordiales, partiendo de que su fundamento se encuentra en el lenguaje en 
cuanto esquema de funcionamiento del sentido otorgado desde el deseo del Otro.

La estructuración, así, implica elementos irreductibles. Sin estos no es posible la fundación de un sujeto provisto de palabra. Los elementos de los que hablamos son los siguientes: el Otro materno, sus cuidados, la respuesta en forma de demanda deseo en una relación en la que el neonato constituye las primeras realizaciones para la pulsión, a la vez que las primeras insatisfacciones, faltas de placer, cifradas de manera real, simbólica e imaginaria que constituyen los objetos de deseo (Lacan, 2004), y la posterior caída del objeto, designado en Lacan como objeto a (Lacan, 2009). La dimensión de la experiencia queda reducida a la cuestión de la manera como la estructura está fundada y cómo esta opera, eficaz o no, en las redes del lenguaje para el sujeto.

Así, el elemento crucial es el significante de la falta del Otro materno, significante que, susceptible de las operaciones y funciones del lenguaje, ordena los demás significantes para que estos a su vez, cuando sean requeridos, hablen en el sujeto sobre la relación que este mantiene con el sentido de su discurso, su verdad de goce y de deseo, por medio de su realización sintomática proyectada desde el fantasma ${ }^{2}$.

De esto podemos deducir que los elementos necesarios para la estructuración subjetiva no son equivalentes a la estructura, lo cual quiere decir que el orden simbólico, prevalente en el mundo humano, no se puede confundir con "el lenguaje" ni con la estructura en cuanto tal. Tampoco se puede confundir con la noción de 'palabra' en la conceptualización realizada por Lacan (2000). Este orden, cultural, aporta las posibilidades de hacer con el objeto, así como las premisas para la comprensión entre seres hablantes a partir del isomorfismo de la ley deseo, por lo cual su vehículo es el lenguaje, poseedor de una estructura en la que existen mínimos de exigencia y condiciones sin las que la creación se hace delirante.

Es evidente en la psicosis, por ejemplo, en la que el sujeto acude precisamente a lo simbólico, pero no quiere decir eso que su estructura corresponda con la isomórfica del compleo de Edipo, lo cual produce la perplejidad del sujeto aquejado por la alucinación o el delirio,

2 En francés fantasme, concepto que indica en el discurso lacaniano la categoría equivalente a fantasía en la obra freudiana. al tiempo que angustia a los de su entorno. Los modos de estructuración no son equivalentes ni referidos a los mismos elementos porque la psicosis realiza una estructura diferente de la neurosis, por lo cual la última es una estructura que hace permanente referencia al significante nombre del padre para signar en él lo que responde del deseo materno. En la psicosis esto no está presente. Si bien no se encuentra anclaje al nombre del padre, es posible que sea a otros significantes, y que la construcción de la realidad, del cuerpo y de las relaciones libidinales se vean afectadas por ello, por lo menos frente a la manera regular que hace todo aquel aquejado de neurosis. El asunto es poder pensar en la estructura como ese juego de interrelaciones, de causas y efectos, frente a la presencia o rotunda ausencia de componentes significantes, lo cual permitirá, en el caso de nosotros, interesados en la práctica analítica, claridad respecto del proceso de la cura, de la dirección del tratamiento.

Para concluir, queda en apertura la pregunta por si el sujeto del inconsciente es estructura, si es un elemento de una estructura o si es producto de la estructura.

\section{EL SUJETO DEL INCONSCIENTE Y SU ESTATUTO FRENTE A LA ESTRUCTURA}

No existe equivalencia entre el concepto 'sujeto del inconsciente' con algún concepto propuesto fuera de la conceptualización psicoanalítica. La idea de individuo, tal vez una de las más usadas para hablar y escribir, resulta inapropiada por su carácter indivisible. Difucultades similares comporta la palabra personalidad, por su carácter imaginario en cuanto semblante y máscara del yo. La diferencia estriba en que el sujeto del inconsciente se revela justo en la falla, ahí, en los fenómenos fallidos logrados del funcionamiento de lo que está estructurado como lenguaje, en las formaciones del inconsciente, determinadas por la cuestión del significante de la falta del Otro materno y asociadas a este en la construcción fantasmática de satisfacción del sujeto.

Por lo tanto, el sujeto no es fenómeno en sí mismo, se revela ahí donde hace parte de una cadena de significantes y de una relación con el Otro. Es producto solo a causa de ser escuchado y de responder frente a lo que se revela en el lapsus, en el síntoma, en el acto fallido, en el sueño. Por ejemplo, frente a la inconformidad
Estructura

y sujeto del

inconsciente:

la ficción de lo simbólico 
permanente del humano con su objeto de satisfacción, dado que este no es natural, pasa por las redes del lenguaje y ahí sufre toda serie de transformaciones para que se establezcan maneras subjetivas de apropiación de la experiencia. Ahí es cuando el sueño deforma o condensa, mezcla y confunde, todo para que el sujeto del inconsciente logre una satisfacción no admitida por el yo. Y justo eso angustia. Por eso, el soñante se despierta con una sensación de ambivalencia frente al contenido que su extimidad le ha mostrado.

El orden simbólico se encarga de trazar las condiciones de traducción y exclusión de lo real para un sujeto, con lo cual genera bordes susceptibles de ser tocados en uno u otro registro, que resultan fuente de conflicto para la fisiología del organismo y de su acaecer psíquico. Por esto para el ser humano las cuestiones del cuerpo dejan de ser una percepción de un sistema de órganos que poseemos para convertirse en un cuerpo idealizado desde la erotización materna y narcisista, con las vicisitudes, aciertos y traumatismos que esto conlleva. Este hecho fue en el que Lacan insistió respecto de que el cuerpo es un imaginario tejido con palabras (Lacan, 2000). Todas estas son las condiciones que introducen entonces al neonato en la cultura y que lo direccionan con elementos para sus posteriores elecciones, permitiéndose dicha escogencia a partir de las estructuras más viables para su satisfacción, con lo cual se generan siempre relaciones de compromiso entre instancias que indican el placer y su restricción.

En consecuencia, las estructuras preexisten al sujeto en cuanto tal, y ellas favorecen su estructuración, pero también existe una elección de objeto y de modos de vérselas con este, con el deseo, con el síntoma y con el goce, que comprometen un funcionamiento estructural del sujeto, es decir, al sujeto como estructura: neurosis, perversión y psicosis.

Las diferentes modalidades siempre son para el deseo del Otro y la particular posición ofrecida por este al sujeto en estructuración. Serán la base para la posterior elección y sedimentación de la estructura subjetiva, la cual denotará una posición discursiva o fuera del discurso en cuanto relación o rechazo del semejante.

Así, el sujeto del inconsciente se constituye en producto de la estructura primordial, susceptible de análisis en con las formaciones del inconsciente la falla estructural insalvable de cada ser hablante, debido a la ficción (Gerber, 1997) realizada en el acto de apalabrar lo real y lo imaginario en el ser humano.

\section{REFERENCIAS BIBLIOGRÁFICAS}

1. Boudon, R. (1972). Para qué sirve la noción de estructura. Madrid: Aguilar.

2. Saussure, F. de (1945). Curso de lingüistica general. Buenos Aires: Losada.

3. Freud, S. (1895/2004). Proyecto de psicología para neurólogos. En Obras completas (t. I). Buenos Aires: Amorrortu.

4. Freud, S. (1893-1895/2005). Estudios sobre la histeria. En Obras completas (t. II). Buenos Aires: Amorrortu.

5. Freud, S. (1895/2004). Las neuropsicosis de defensa. En Obras completas (t. 3). Buenos Aires: Amorrortu.

6. Freud, S. (1895/2004). Nuevas puntualizaciones sobre las neuropsicosis de defensa. En Obras completas (t. III). Buenos Aires: Amorrortu.

7. Freud, S. (1898/2004). El mecanismo psíquico de la desmemoria. En Obras completas (t. III). Buenos Aires: Amorrortu.

8. Freud, S. (1899/2004). Los recuerdos encubridores. En Obras completas (t. III). Buenos Aires: Amorrortu.

9. Freud, S. (1900/2004). La interpretación de los sueños. En Obras completas (t. IV). Buenos Aires: Amorrortu.

10. Freud, S. (1901/2005). Psicopatología de la vida cotidiana. En Obras completas (t. VI). Buenos Aires: Amorrortu.

11. Freud, S. (1915/2004). Lo inconsciente. En Obras completas (t. XIV). Buenos Aires: Amorrortu.

12. Freud, S. (1923/2004). El yo y el ello. En Obras completas (t. XIX). Buenos Aires: Amorrortu.

13. Gerber, D. (1997). Ficciones de verdad. En El laberinto de las estructuras (vol. I). México: Siglo XXI.

14. Lacan, J. (2000). El estadio del espejo como formador de la función del yo [Je] tal como se nos revela en la experiencia psicoanalítica. En Escritos 1. México: Siglo XXI. 
15. Lacan, J. (2000). Función y campo de la palabra y del lenguaje en psicoanálisis. En Escritos 1. México: Siglo XXI.

$\mid \begin{aligned} & \text { Estructura } \\ & \text { y sujeto del } \\ & \text { inconsciente: }\end{aligned}$

16. Lacan, J. (2000). La instancia de la letra en el inconsciente o la razón después de Freud. En Escritos 1. México: Siglo XXI.

17. Lacan, J. (2004). El seminario. Libro 4. La relación de objeto. Buenos Aires: Paidós.

18. Lacan, J. (2009). El seminario. Libro 10. La angustia. Buenos Aires: Paidós. la ficción de lo

simbólico 